\title{
Perfiles de rechazo escolar: identificación y comparación entre adolescentes ecuatorianos y chilenos
}

\author{
School refusal behavior profiles: identification and comparison
}

between ecuadorian and chilean adolescents

Carolina Gonzálvez ${ }^{1}$, Cándido J. Inglés ${ }^{2}$, María Vicent ${ }^{3}$,

Carlos M. Calderón-Guevara ${ }^{4}$, Nelly Lagos-San Martín ${ }^{5}$, Ricardo Sanmartín ${ }^{6}$

y José Manuel García-Fernández ${ }^{7}$

${ }^{1}$ Doctora en Investigación Educativa. Profesora Contratada en el Departamento de Psicología

Evolutiva y Didáctica, Facultad de Educación, Universidad de Alicante, España.

E-mail: carolina.gonzalvez@ua.es

${ }^{2}$ Doctor en Psicología. Profesor Titular en el Departamento de Psicología de la Salud, Facultad de Psicología, Universidad Miguel Hernández de Elche, España. E-mail: cjingles@umh.es

${ }^{3}$ Doctora en Investigación Educativa. Profesora Ayudante en el Departamento de Psicología Evo-

lutiva y Didáctica, Facultad de Educación, Universidad de Alicante, España.

E-mail: maria.vicent@ua.es

${ }^{4}$ Doctor en Investigación Educativa. Profesor Titular en la Facultad de Filosofía, Literatura y

Ciencias de la Educación, Universidad Central de Ecuador, Ecuador.

E-mail: chekalberto@yahoo.com

${ }^{5}$ Doctora en Investigación Educativa. Profesora Titular en el Departamento de Ciencias de la

Educación de la Universidad del Bío-Bío, Chillán, Chile. E-mail: nlagos@ubiobio.cl

${ }^{6}$ Estudiante de Doctorado. Profesor Ayudante en el Departamento de Psicología Evolutiva y Di-

dáctica de la Universidad de Alicante, España. E-mail: ricardo.sanmartin@ua.es

${ }^{7}$ Doctor en Psicología. Profesor Titular en el Departamento de Psicología Evolutiva y Didáctica de la Universidad de Alicante, España. E-mail: josemagf@ua.es

Esta investigación ha sido financiada por el proyecto FONDECYT (11160040) de CONICYT (Chile) y el grupo de investigación GIDECAP (GI1608320/EF). Han colaborado investigadores procedentes de dos universidades españolas (Universidad de Alicante y Universidad Miguel Hernández de Elche), una ecuatoriana (Universidad Central de Ecuador) y una chilena (Universidad del Bío-Bío).

\section{Resumen}

La relevancia psicológica, social y educativa del presente estudio reside en la escasez de trabajos previos que hayan evaluado el rechazo escolar en Latinoamérica y las elevadas tasas de deserción a las que se enfrentan en esta región. El objetivo de esta investigación consistió en examinar las diferencias en las puntuaciones medias de rechazo escolar y la formación de perfiles en adolescentes ecuatorianos y chilenos. Un total de 4266 estudiantes, entre 13 y 17 años de edad, procedentes de Ecuador $(M=14.83$; $S D=$ 1.86) y Chile $(M=15.23 ; S D=1.26)$ fueron seleccionados mediante un muestreo aleatorio 
por conglomerados. El instrumento empleado para evaluar el rechazo escolar fue la School Refusal Assessment Scale-Revised. Las puntuaciones medias de rechazo escolar para Ecuador y Chile revelaron diferencias estadísticamente significativas de pequeña magnitud. Entre los estudiantes chilenos se registraron puntuaciones medias más altas de rechazo escolar, con el fin de captar la atención de sus seres queridos y obtener reforzadores tangibles externos a la escuela, en comparación con sus iguales procedentes de Ecuador. Los análisis de conglomerados confirmaron cuatro perfiles de rechazo escolar en ambos países: Rechazo Escolar Bajo, Rechazo Escolar Alto, Rechazo Escolar Ansioso y Rechazo Escolar Absentista. A pesar de que los adolescentes chilenos obtuvieron puntuaciones medias significativamente más altas que los ecuatorianos en algunas dimensiones del rechazo escolar, los resultados revelaron una pequeña variabilidad entre los perfiles de rechazo escolar encontrados en ambos países. Los resultados obtenidos se discuten atendiendo a las características culturales definitorias de cada uno de estos países.

Palabras clave: Rechazo escolar; Chile; Ecuador; Adolescentes; Perfiles.

\section{Abstract}

School refusal behavior is defined as any child or youth's difficulty to attend classes or to remain in the school. This behavior could be based or not on anxiety and prevalence rates from 5 to $28 \%$ if it is considered any type of rejection towards the school. Adolescents demonstrating school refusal behaviors are very likely to demonstrate poorer academic performance, more stress, behavior problems and family conflicts. The psychological, social and educational relevance of the present study is due to the scarce of previous studies that have evaluated the school refusal behavior in Latin America and the high dropout rates that they face in this region. This study is focused on two countries, Ecuador and Chile, in order to identify possible differences regarding school refusal behavior scores between adolescents in both countries. The aim of this investigation was to examine the mean differences scores on school refusal behavior and the formation of school refusal behavior profiles in Ecuadorian and Chilean adolescents to determine whether these results differ from one another based on the geographical origin. In this study participated a total of 4266 students aged between 13 and 17 years old from Ecuador $(M$ $=14.83, S D=1.86 ; \mathrm{N}=1588)$ and Chile $(M=$ 15.23; $S D=1.26 ; \mathrm{N}=2678$ ). These students were selected by cluster random sampling. The measure used to assess the school refusal behavior was the School Refusal Assessment Scale-Revised (SRAS-R). The SRAS-R is a self-report measure that assesses the relative strength of four proposed functions, or maintaining variables, of school refusal behavior: Factor I. Avoidance of school-based stimuli that provoke Negative Affectivity; Factor II. Escape from aversive Social/Evaluative situations at school; Factor III. Pursuit of Attention from Significant others; and Factor IV. Pursuit of Tangible Reinforcement outside of school. The SRAS-R was administered during the school day (a session of approximately 20 minutes) in groups who completed the scale anonymously in accordance with the ethical standards and also emphasizing the voluntary nature of the test. Regarding the results, mean differences scores on school refusal behavior revealed statistically significant differences of small magnitude with Chilean students reaching higher mean scores in school refusal behavior in order to pursuit the attention from significant others and to obtain tangible reinforces external to the school, in comparison with their equals from Ecuador. Cluster analysis confirmed four school refusal profiles in both countries: Low School Refusal Behavior (characterized by low school refusal behavior scores for the four factors of the SRAS-R), High School Refusal Behavior (characterized by high school refusal behavior scores for the four factors of the SRAS- 
R), Anxious School Refusal Behavior (profile that combines high scores on the first three factors of the SRAS-R and moderate scores for the fourth) and Absentee School Refusal Behavior (profile that combines moderate levels for the first three factors of the SRAS-R but high in the fourth factor). The results revealed a small variability between the school refusal behavior profiles across the countries. However, Chilean adolescents scored significantly higher than Ecuadorians in school refusal based on the search for tangible external reinforcements. The results of this research supported a low variability of the findings according to the country. The generalization of these results supports the transcultural validity of the SRAS-R and emphasizes that Ecuador and Chile share a similar situation regarding school refusal behavior. Some limitations of this work are considered, and they are proposed as future lines of research. To conclude, the results are discussed taking into account the defining cultural characteristics of each country.

Keywords: School refusal behavior; Chile; Ecuador; Adolescents; Profiles.

\section{Introducción}

El rechazo escolar se refiere a la negativa o dificultad persistente de un estudiante para asistir o permanecer en la escuela (Kearney, 2016). En sus inicios, países como Estados Unidos, Reino Unido, Canadá y Australia fueron considerados pioneros en la investigación del rechazo a la escuela (Kearney, 2008). Sin embargo, la repercusión social y educativa de esta problemática hace que en los últimos años su investigación haya alcanzado nuevos contextos culturales (véase para una revisión Inglés, Gonzálvez, García-Fernández, Vicent y Martínez-Monteagudo, 2015).

La escolarización durante la primera infancia y la adolescencia, que implica la asistencia regular al centro educativo, debe de ser un derecho de todos los estudiantes, así como una necesidad para la población infantil y juvenil al constituir un factor de protección ante posibles riesgos sociales (Gonzálvez, Inglés y García-Fernández, 2018). Investigaciones previas señalan la relación identificada entre los estudiantes que presentan problemas o dificultades para asistir a la escuela y su vinculación con peores resultados en el rendimiento académico (Barry, Chaney y Chaney, 2010; Thornton, Darmody y McCoy, 2013; Yahaya et al., 2010), mayor comorbilidad con trastornos de ansiedad y depresión (Fernández-Sogorb et al., 2018; Kearney y Albano, 2004), o el incremento de riesgo de presentar conductas externalizantes tales como el consumo de sustancias estupefacientes $\mathrm{y} / \mathrm{o}$ alcohol, desobediencia o conducta agresiva (Maynard, Salas-Wright, Vaughn y Peters, 2012; Vicent, Inglés, Sanmartín, Gonzálvez y García-Fernández, 2018).

En Latinoamérica, a pesar del incremento sostenido que se produce en el porcentaje de adolescentes que abandonan de manera temprana el sistema educativo (SITEAL, 2010), son escasas las investigaciones que se han centrado en analizar el rechazo a la escuela. En este campo, la deserción escolar sigue siendo un problema en esta región (Palomar y Victorio, 2016), sobre todo durante la etapa de escolaridad secundaria, en la que apenas ha disminuido el promedio de su prevalencia de un $16.9 \%$ en 2000 a un $15.5 \%$ en 2012 (Moreno, 2013). Mientras que en Ecuador no se han encontrado estudios previos sobre rechazo escolar, investigaciones recientes reportan diferencias en rechazo a la escuela en función del sexo y la edad en adolescentes chilenos (García-Fernández, Inglés, Lagos et al., 2016).

A estos datos cabe sumar el interés en Latinoamérica por reducir el número de estudiantes que participan en actividades laborales, ya sea en el hogar o en contextos fuera de casa, durante su escolarización obligatoria. Investigaciones alertan de la repercusión negativa y perjudicial que tiene un empleo en estas edades y cómo influye en el rendimiento académico (Post, 2011). Esta problemática, 
en otros contextos como Europa o Norte América, se comenzó a debatir y luchar por su erradicación en el sector industrial a lo largo del siglo XIX (Pedraza, 2007).La consideración de la infancia, como una etapa fundamental del desarrollo que requiere protección y cuidado, queda reflejado en la Convención de los Derechos del Niño de 1989 siendo un propósito a alcanzar a nivel mundial.

Oyserman, Coon y Kemmelmeier (2002) señalan que para estudiar con precisión la variación transcultural de un fenómeno en Latinoamérica, es preciso realizar investigaciones que incluyan poblaciones de distintos países latinoamericanos y así, poder generalizar acerca de las diferencias y semejanzas culturales con otras regiones del mundo. En los últimos años, a la lista de países considerados líderes en la investigación sobre rechazo escolar durante la primera década del siglo XX (Estados Unidos, Reino Unido, Canadá $\mathrm{y}$ Australia),se han incorporado otros países europeos y asiáticos, tales como España, Holanda o Japón. A pesar de estos avances, predominan las investigaciones realizadas con muestra estadounidense y europea (García-Fernández, Inglés, Gonzálvez et al., 2016), culturas que tienden hacia el individualismo, en contraste con la escasez de trabajos sobre rechazo escolar en Latinoamérica, con una población que tiende al colectivismo (Estrada y Terpstra, 2014).Los términos individualismo y colectivismo son dos términos utilizados para comparar las características culturales de dos sociedades diferentes. El individualismo se refiere a sociedades que priorizan las necesidades y metas de uno mismo, considerándose individuos bastante independientes de su grupo social. Por otro lado, el colectivismo se vincula a sociedades en las que las personas se consideran parte un grupo social, por lo que han de cumplir una serie de hábitos, tradiciones o normas que el propio grupo social (familia, tribu, etc.) establece (Estrada y Terpstra, 2014).Si bien trabajos previos señalan un cierto equilibrio respecto a las tasas de rechazo escolar a nivel racial y sociocultural (Kearney y Bates, 2005), se requiere ampliar la investigación en este ámbito para determinar en qué situaciones las diferencias entre grupos de distintos países o estamentos socioeconómicos distintos, pueden ser significativas (Lyon, 2010).

Abordar la problemática del rechazo escolar parte de la necesidad de conocer con precisión las características que definen a los distintos tipos de estudiantes que rechazan la escuela. Esta tarea resulta ardua y compleja debido a las múltiples causas que pueden justificar el comportamiento de rechazo escolar (Gonzálvez et al., 2018). Es por ello que la intervención ante una población tan heterogénea partirá de la necesidad de diseñar estrategias multimodales adaptadas a las características individuales y al contexto social y cultural de los estudiantes. Existen trabajos que han comprobado la funcionalidad de la terapia cognitivo conductual ante casos de rechazo a la escuela (Kearney y Albano, 2007; King et al., 1998), incluso guías específicas que formulan pautas de intervención para el tratamiento del rechazo escolar (Blagg, 1987). Sin embargo, no se han encontrado estudios de carácter preventivo sobre el diseño de programas para atender las necesidades emocionales y otras variables psicológicas o del entorno social y familiar que afectan a estos estudiantes.

Con el fin de lidiar con esta problemática, la identificación de distintos grupos o perfiles de estudiantes que rechazan la escuela permitiría disponer de una información más precisa y novedosa acerca de las características que definen a estos estudiantes. A pesar de que resultan escasas las investigaciones realizadas sobre este tema, Dube y Orpinas (2009) llevaron a cabo la identificación de perfiles de rechazo a la escuela en una muestra no clínica de 99 estudiantes americanos entre 8 y 15 años de edad $(M=12.5 ; S D=1.38)$. Los resultados revelaron tres grupos de estudiantes que rechazaban la escuela. Uno de ellos caracterizado por negarse a asistir a la escuela por reforzamiento negativo y positivo (principalmente basado en la manifestación de síntomas ansiógenos ante 
la obligación de asistir la escuela); otro grupo caracterizado por estudiantes que rechazan la escuela con el fin de alcanzar reforzadores positivos (quedarse en casa o en el parque realizando actividades de ocio y tiempo libre) y un grupo de estudiantes con bajas puntuaciones en rechazo escolar.

Con el fin de solventar la carencia de estudios transculturales y aportar una nueva línea de investigación sobre la identificación de perfiles en grupos de estudiantes que rechazan la escuela, el presente estudio tiene como objetivos comprobar si existen diferencias en las puntuaciones medias de rechazo escolar según la nacionalidad ecuatoriana o chilena de los estudiantes, e identificar los perfiles de rechazo escolar y contrastar su formación en función de la procedencia ecuatoriana o chilena de los participantes. Se espera que no existan diferencias significativas en las puntuaciones medias de rechazo escolar y en la formación de los grupos de perfiles entre ambos países, en consonancia con estudios anteriores que revelan un equilibrio del rechazo a la escuela a nivel racial y sociocultural (Kearney y Bates, 2005).

\section{Método}

\section{Participantes}

La selección de dos muestras procedentes de Ecuador y Chile se basó en los siguientes criterios: a) geográficos, ambos son países hispanohablantes, latinoamericanos y ubicados en la costa del Océano Pacífico; b) demográficos, ambos tienen poblaciones de tamaño similar (16.6 millones en Ecuador y 18.3 millones en Chile); y c) políticos, ambos son sistemas democráticos.

La muestra de este estudio se reclutó mediante un muestro aleatorio por conglomerados y estuvo compuesta por 1588 estudiantes procedentes de Ecuador (54.4\% varones) y 2678 de Chile (43.7\% varones). Concretamente, los estudiantes procedían de la ciudad de Quito en Ecuador y de siete comunas de la provincia Nuble en Chile. El rango de edad oscilaba entre los 13 y 17 años en ambos grupos $\left(M_{\text {Ecuador }}=14.83, S D=1.86\right.$; $\left.M_{\text {Chile }}=15.23, S D=1.26\right)$. La prueba Chi Cuadrado reveló que no existían diferencias significativas en función del sexo y la edad para los dos grupos (Ecuador: $\chi^{2}=3.35, p=$ .34; Chile: $\left.\chi^{2}=8.23, p=.08\right)$.

\section{Procedimiento}

Los participantes cumplimentaron el cuestionario en horario escolar en una sesión de 20 minutos, siempre con la presencia de al menos un miembro del grupo de investigación. Previamente, se contó con el consentimiento firmado por escrito de los padres o tutores legales.

\section{Instrumento}

La School Refusal Assessment Scale-Revised (SRAS-R; Kearney, 2002) evalúa el rechazo escolar en niños y adolescentes entre 8-17 años en base a cuatro factores explicativos. El primer factor, I. Evitar la afectividad negativa que provocan estímulos o situaciones relacionadas con el ámbito escolar, se ha asociado a estudiantes cuyo rechazo a la escuela se asocia a síntomas ansiógenos, súplicas constantes, quejas somáticas, trastornos de ansiedad y depresión (Kearney y Albano, 2004; Kearney, Lemos y Silverman, 2006). Un ejemplo de los ítems que componen este factor es “¿Cuántas veces tratas de no ir a la escuela porque si vas te sentirás triste o deprimido?".

El segundo factor, II. Escapar de la aversión social y/o situaciones de evaluación en la escuela, se relaciona con aquellos estudiantes que presentan dificultades en el plano social y sufren ante la realización de pruebas de evaluación. Los estudiantes que justifican su rechazo escolar por esta causa se vinculan a puntuaciones más altas de ansiedad social, comportamientos retraídos y trastorno de ansiedad generalizada (Kearney y Albano, 2004). Un ejemplo de ítem de este factor es 
“Cuántas veces tratas de no ir a la escuela porque te resulta difícil hablar con los otros chicos(as)?".

El tercer factor, III. Búsqueda de la atención de otras personas significativas, se asocia a aquellos estudiantes que pretenden captar la atención de sus seres queridos con no asistiendo a la escuela para poder quedarse con ellos. Este factor ha mostrado correlaciones estadísticamente significativas y positivas con el trastorno de ansiedad por separación y el trastorno negativista desafiante (Kearney y Albano, 2004). Un ejemplo de los ítems que componen este factor es “¿Cuántas veces piensas en tus padres o en tu familia cuando estás en la escuela?".

Por último, el cuarto factor, IV. Búsqueda de refuerzos tangibles fuera del ámbito escolar, se caracteriza por estudiantes que basan su rechazo escolar en el deseo por realizar otras actividades de carácter más recreativo fuera de la escuela, tales como quedarse en casa jugando con videojuegos o en el parque con otros compañeros. Los estudiantes que justifican su rechazo por esta razón, suelen tener edades más avanzadas, adolescentes, y se han vinculado con el trastorno negativista desafiante (Kearney y Albano, 2004). Un ejemplo de ítem de este factor es "Cuando no estás en la escuela durante la semana, ¿cuánto disfrutas haciendo cosas distintas (por ejemplo, estar con amigos, salir)?’.

La SRAS-R está compuesta por 24 ítems con una escala de respuesta tipo Likert de 7 puntos (0: nunca; 6: siempre). Sus adecuadas propiedades psicométricas han sido apoyadas tanto por estudios realizados en muestra clínica (Alemania: Walter, Bialy, Wirth y Doepfner, 2017; Estados Unidos: Kearney, 2002, 2006; Haight et al. 2011; Holanda: Heyne, Vreeke, Maric, Boelens y Van Widenfelt, 2016) como en muestra comunitaria (España: Gonzálvez et al., 2016; Estados Unidos: Lyon, 2010; Inglaterra: Richards y Hadwin, 2011; Turquía: Seçer, 2014). En este estudio los coeficientes de consistencia interna fueron $.74, .72, .81$ y .71 para cada uno de los cuatro factores, respectivamente, en Ecuador, y .74, .72, .80 y .71 en el caso de Chile.

\section{Análisis estadísticos}

Usando el paquete estadístico SPSS 24, se determinaron las puntuaciones medias en rechazo escolar para las dos nacionalidades mediante análisis de varianza (ANOVA). Se utilizaron pruebas post hoc (método de Scheffé) para identificar entre qué grupos existen diferencias incluyendo el índice $d$ para valorar el tamaño del efecto de las diferencias halladas (tamaño del efecto pequeño $.20 \leq d$ $\leq .49$, moderado $.50 \leq d \leq .79$ y alto $d \geq .80$ ) (Cohen, 1988).

Para establecer los perfiles de rechazo escolar, se utilizó el método no jerárquico quick cluster analysis. Se consideraron niveles moderados las puntuaciones $Z$ entre $-.5 \mathrm{y}+.5$, niveles bajos, -.5 , y niveles altos +.5 (Inglés et al., 2016). Previamente al análisis de conglomerados, se estandarizaron las puntuaciones y la elección del número de clusters se realizó tomando como criterios maximizar las diferencias intergrupo y valorando los referentes teóricos y el significado psicológico de cada uno de los grupos que representan los distintos perfiles de rechazo escolar.

\section{Resultados}

\section{Diferencia de medias}

En la Tabla 1 se comparan las puntuaciones medias en rechazo escolar para Ecuador y Chile. Los resultados revelan diferencias estadísticamente significativas para el tercer y cuarto factor de la SRAS-R (III. Captar la atención y IV. Obtener refuerzos tangibles). Concretamente, son los estudiantes chilenos quienes reportaron puntuaciones medias más altas en rechazo escolar para estos factores que sus iguales procedentes de Ecuador. No obstante, atendiendo al índice $d$ tan solo resultaría significativa y de tamaño pequeño la diferencia hallada para el cuarto factor $(d=.29)$. 
Tabla 1

Diferencias en las puntuaciones medias de rechazo escolar en Ecuador y Chile.

\begin{tabular}{c|c|c|c|c|c|c|c|c}
\multirow{2}{*}{$\begin{array}{c}\text { Rechazo } \\
\text { escolar }\end{array}$} & $\begin{array}{c}\text { Prueba } \\
\text { Levene }\end{array}$ & \multicolumn{2}{|c|}{ Ecuador } & \multicolumn{3}{c|}{ Chile } & \multicolumn{3}{c}{$\begin{array}{c}\text { Significación estadística y } \\
\text { magnitud diferencias }\end{array}$} \\
& $F$ & $M$ & $D E$ & $M$ & $D E$ & $t$ & $g . l$. & $d$ \\
\hline FI SRAS-R & $7.95^{*}$ & 14.73 & 6.31 & 14.89 & 6.54 & -.82 & 3918.90 & - \\
FII SRAS-R & .16 & 13.36 & 5.73 & 13.09 & 5.85 & 1.53 & 4461 & - \\
FIII SRAS-R & 2.05 & 16.87 & 7.15 & 17.65 & 7.28 & $-3.50^{* *}$ & 4461 & .11 \\
FIV SRAS-R & 2.24 & 20.81 & 7.26 & 22.85 & 6.97 & $-9.41^{* *}$ & 4460 & .29
\end{tabular}

Notas: FI SRAS-R: rechazo a evitar emociones negativas; FII SRAS-R: rechazo a escapar de la aversión social o situaciones de evaluación; FIII SRAS-R: rechazo para llamar la atención; FIV SRAS-R: rechazo para obtener recompensas fuera de la escuela. ${ }^{*} p<.01 .{ }^{*} p<.001$.

\section{Perfiles de rechazo escolar y datos de porcentaje}

La figura 1 representa los cuatro perfiles de rechazo escolar resultantes en Ecuador tras la combinación de altas, bajas y moderadas puntuaciones en los cuatro factores que componen la SRAS-R.En este país, el primer clúster estuvo formado por 658 sujetos $(36.8$ $\%$ ) caracterizado por bajas puntuaciones en rechazo escolar para los cuatro factores de la SRAS-R. Este grupo se denominó Rechazo Escolar Bajo. El segundo grupo estuvo compuesto por 164 participantes $(9.2 \%)$ quienes presentaron altas puntuaciones en los cuatro factores del rechazo escolar. Este grupo recibió el nombre de Rechazo Escolar Alto. El siguiente grupo estuvo integrado por 418 sujetos $(23.4 \%)$ y revela un perfil que combina niveles moderados para los tres primeros factores de la SRAS-R, pero altos en el cuarto factor. Teniendo en consideración que el cuarto factor de la SRAS-R se asocia comúnmente al absentismo escolar,tipo de ausencia no basado en la ansiedad y que suele llevarse a cabo sin el consentimiento de los padres, se consideró apropiado designar a este grupo como Rechazo Escolar Absentista. Por último, el cuarto grupo estuvo formado por 546 participantes $(30.6 \%)$ que obtuvieron altas puntuaciones en los tres primeros factores de la SRAS-R y moderadas para el cuarto. En este caso se decidió nombrar a este grupo como Rechazo Escolar Ansioso debido a que los tres primeros factores de la SRAS-R sí han mostrado vinculación con la manifestación de síntomas ansiógenos, así como trastornos de ansiedad y depresión.

Resultados similares se obtuvieron en Chile, por lo que se utilizaron los mismos términos empleados en Ecuador para denominar a los perfiles (véase Figura 2). En este país el primer grupo estuvo compuesto por 1040 participantes (38.9\%) y se corresponde con el perfil de Rechazo Escolar Bajo. El segundo clúster estuvo formado por 362 sujetos $(13.5 \%)$ quienes presentaron un perfil de Rechazo Escolar Alto y el siguiente grupo estuvo integrado por 631 participantes (23.6 $\%$ ) coincidiendo con un perfil de Rechazo Escolar Absentista. Por último, el cuarto clúster estuvo compuesto por 643 sujetos (24 $\%$ ) quienes obtuvieron puntuaciones altas para los dos primeros factores de la SRAS-R y moderadas para el tercer y cuarto factor. Este grupo se definió como Rechazo Escolar Ansioso. A pesar de que en Ecuador este perfil se caracterizó por altas puntuaciones en los tres primeros factores de la SRAS-R, cabe señalar que de los cuatro factores que conforman este instrumento los dos primeros son los que presentan una mayor vinculación con problemas y trastornos de ansiedad, por lo que se ha decido mantener la misma nomenclatura para este perfil en Chile. 


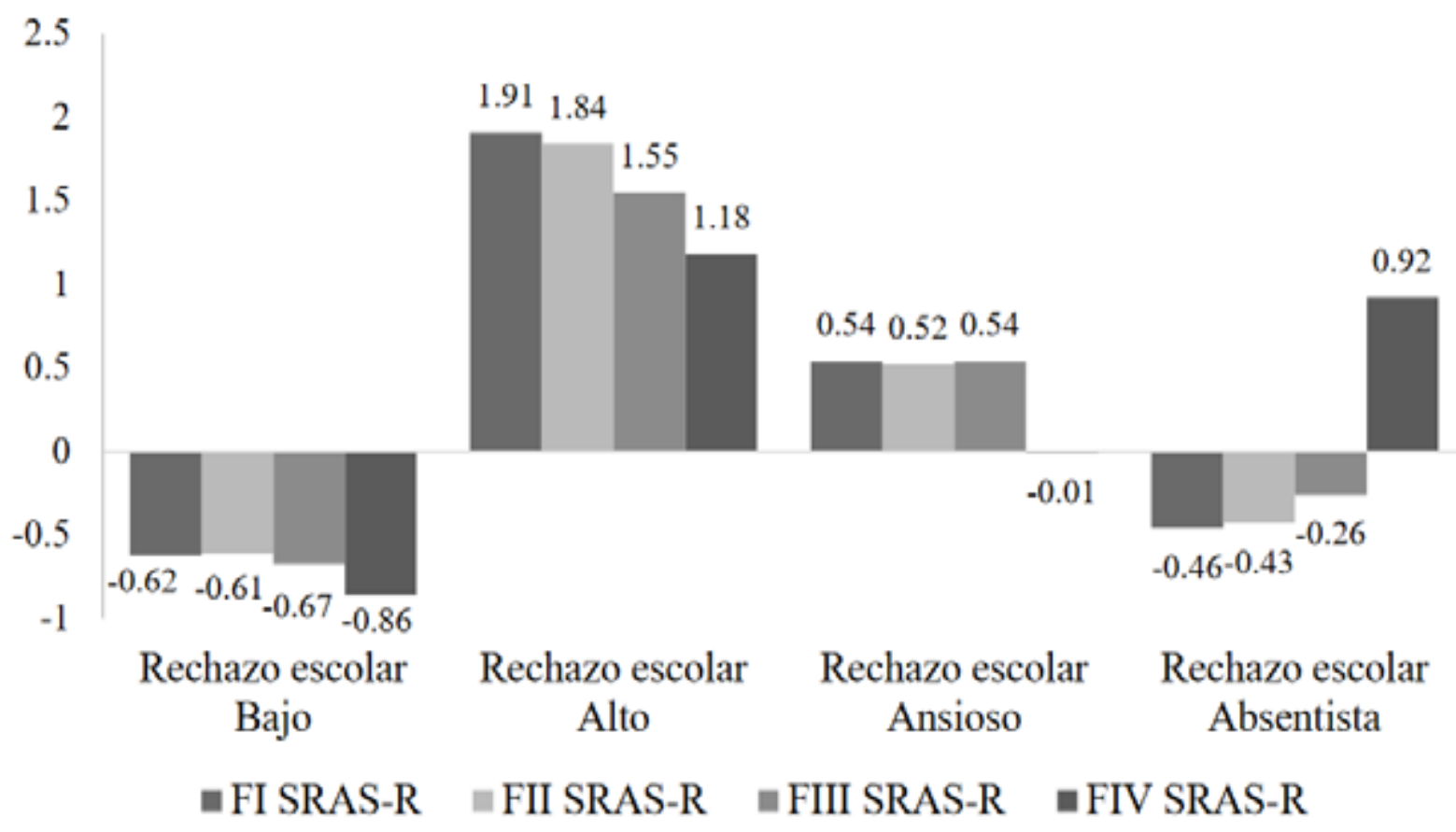

Figura 1. Perfiles de rechazo escolar en Ecuador.

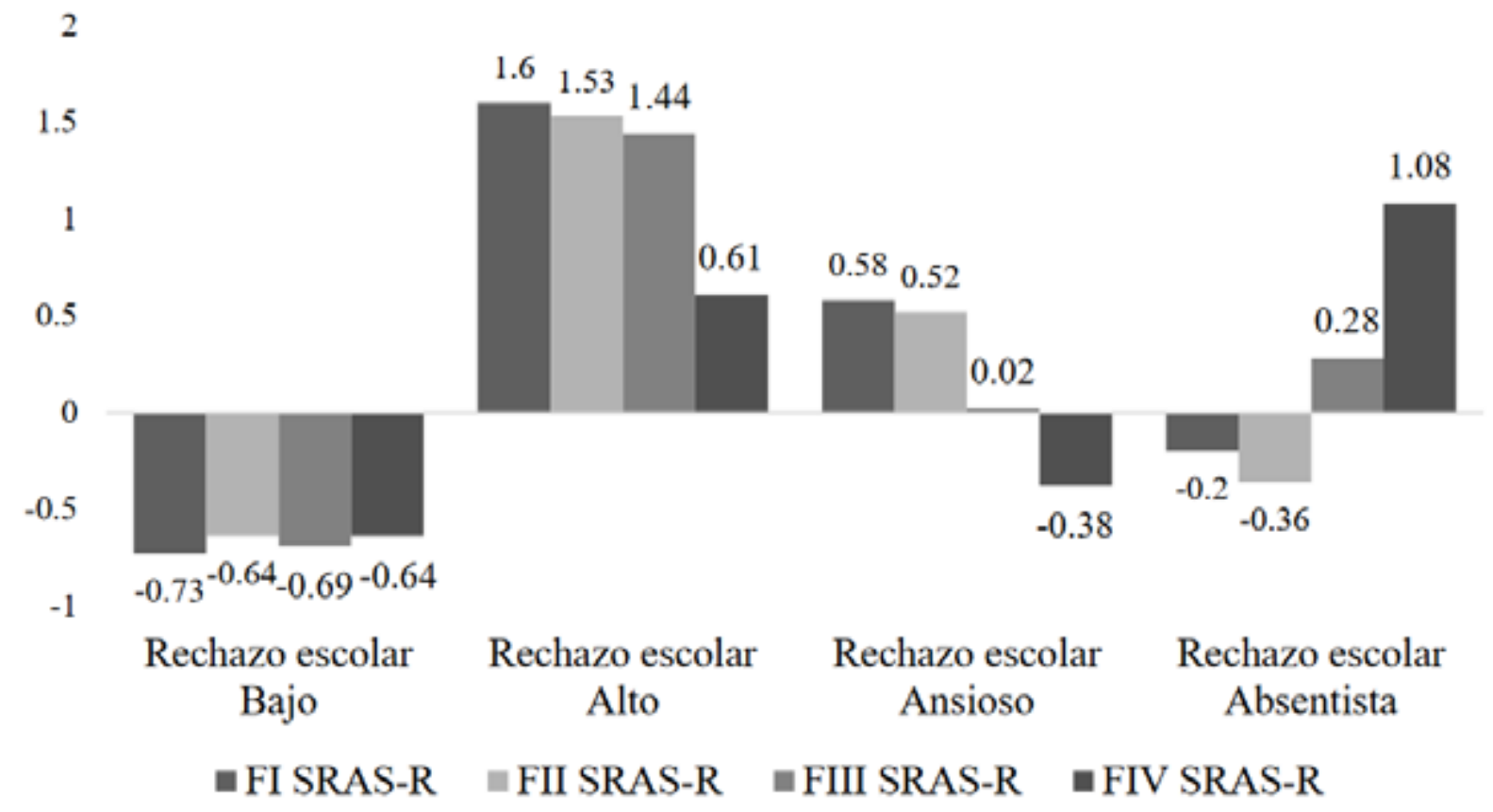

Figura 2. Perfiles de rechazo escolar en Chile.

La Tabla 2 muestra los porcentajes de representatividad, tan solo se hallaron diferencias significativas para el porcentaje de estudiantes con un perfil de Rechazo Escolar Alto que fue superior en Chile, mientras que el grupo de Rechazo Escolar Ansioso fue superior en Ecuador. No obstante, la magnitud de las diferencias no fue relevante $(d=.13$ у .15 , respectivamente). 
Tabla 2

Porcentajes de representatividad de los perfiles de rechazo escolar en Ecuador y Chile.

\begin{tabular}{|c|c|c|c|c|c|}
\hline \multirow{2}{*}{ Perfil de rechazo escolar } & \multirow{2}{*}{$\begin{array}{c}\text { Ecuador } \\
\mathrm{N}=1786(\%)\end{array}$} & \multirow{2}{*}{$\begin{array}{c}\text { Chile } \\
N=2676(\%)\end{array}$} & \multicolumn{3}{|c|}{$\begin{array}{l}\text { Significación estadística } \\
\text { y magnitud diferencias }\end{array}$} \\
\hline & & & $\mathbf{Z}$ & $\mathbf{p}$ & d \\
\hline Rechazo escolar Bajo & 36.8 & 38.9 & -1.41 & n.s. & - \\
\hline Rechazo escolar Alto & 9.2 & 13.5 & -4.36 & $<.001$ & .13 \\
\hline Rechazo escolar Ansioso & 30.6 & 24 & 4.88 & $<.001$ & .15 \\
\hline Rechazo escolar Absentista & 23.4 & 23.6 & -.15 & n.s. & - \\
\hline
\end{tabular}

\section{Discusión}

Desde una perspectiva transcultural y con el fin de determinar si el rechazo escolar tiene el mismo impacto en adolescentes procedentes de distintos países latinoamericanos se desarrolló el presente estudio. Su objetivo consistió en analizar las diferencias en las puntuaciones medias de rechazo escolar y la formación de perfiles en adolescentes de nacionalidad ecuatoriana y chilena.

Los resultados de esta investigación apoyaron la hipótesis inicial al encontrar una escasa variabilidad de los hallazgos en función del país. La generalización de estos resultados permite, por un lado, respaldar la validez transcultural de la SRAS-R y por otro, remarcar que Ecuador y Chile comparten una situación similar en materia de rechazo escolar.

En cuanto al número de perfiles obtenidos, la presente investigación ha identificado cuatro perfiles de estudiantes que rechazan la escuela en comparación con los tres grupos identificados por Dube y Orpinas (2009). A pesar de esta diferencia en cuanto al número de perfiles, los grupos obtenidos son bastante semejantes en los dos estudios. Se ha encontrado un grupo de bajo rechazo escolar que coincide en ambos estudios, un grupo que basa su rechazo a la escuela por reforzamiento positivo y negativo que se corresponde en este trabajo con el grupo de Rechazo Escolar Ansioso y un grupo con rechazo escolar por reforzamiento positivo que se corresponde en este trabajo con el grupo de Rechazo Escolar
Absentista. La principal diferencia es que en este estudio se ha identificado un nuevo perfil con altas puntuaciones en todas las dimensiones de rechazo escolar evaluadas. Estas pequeñas diferencias podrían deberse al tipo de análisis estadísticos aplicados, ya que en el estudio de Dube y Orpinas (2009) la formación de los perfiles se realiza a partir de las puntuaciones medias directas en cada una de las dimensiones del rechazo escolar evaluadas mientras que, en este trabajo se aplica un análisis más sofisticado y preciso como es el análisis de conglomerados. A su vez, la realización de estos estudios se ha desarrollado en distintos países, condición que también podría influir en los resultados alcanzados.

A pesar de que Chile destaca entre los países latinoamericanos por sus logros educativos en materia de escolarización y menores tasas de deserción (Espinoza, González, Cruz, Castillo y Loyola, 2014), en este trabajo los estudiantes chilenos puntuaron más alto que sus iguales ecuatorianos en rechazo escolar basado en la búsqueda de refuerzos tangibles externos. Estos resultados podrían justificarse debido al esfuerzo de las políticas educativas chilenas por alcanzar una escolarización plena durante la etapa de escolaridad secundaria. Consecuencia de este propósito, es la presencia de estudiantes escolarizados que, a pesar de asistir al centro escolar, expresan su negativa a permanecer en la escuela.

Aunque la prevalencia de este tipo de rechazo haya sido menor en Ecuador, este país se enfrenta a dos problemáticas que repercuten sobre el comportamiento de rechazo a 
la escuela: la deserción y el trabajo infantil. Concretamente, Post (2011) advierte que un $6 \%$ de los adolescentes chilenos trabajan fuera de la escuela 4 horas o más, mientras que en Ecuador el porcentaje asciende hasta el $14 \%$. El análisis de esta realidad quedaría incompleto si no se considera la influencia que ejerce el contexto sociocultural sobre estas conductas (Kashima, 2016). La sociedad latinoamericana suele caracterizarse por una cultura colectivista que implica sentimientos de responsabilidad por el bienestar del grupo y enfatiza la importancia de la familia. La suma de estas experiencias culturales y la falta de ingresos económicos en el seno familiar, podrían justificar las tasas de adolescentes que trabajan a la vez que estudian. Esta situación se convierte en grave cuando interfiere negativamente sobre el rendimiento académico y desencadena en el rechazo o abandono escolar (Murrieta, 2016).

En cuanto a los porcentajes que representan los distintos perfiles de rechazo, resulta coherente que en una muestra comunitaria como la de este estudio el grupo con bajas puntuaciones en todas las dimensiones de rechazo escolar fuera el más frecuente tanto en Ecuador como en Chile, con más de un $35 \%$ de representación. No obstante, preocupa que más de un $20 \%$ de los estudiantes en ambos países puedan enmarcarse dentro de un grupo que basa su negativa a asistir a la escuela en síntomas ansiógenos (Rechazo Escolar Ansioso) o bien porque prefieren estar fuera del centro escolar durante el horario lectivo realizando actividades de ocio con otros compañeros o en casa (Rechazo Escolar Absentista). Ante estos casos, se sugieren como medidas de actuación:el diseño y aplicación de programas preventivos dirigidos al control y disminución de reacciones emocionales negativas; la detección temprana de casos de rechazo a escolar, y ofrecer una atención acorde a las características particulares de cada uno de los perfiles de rechazo.

A pesar de estos hallazgos, es necesario mencionar que este estudio posee diversas limitaciones que esperan ser solventadas en futuros trabajos. En primer lugar, se espera que próximas investigaciones amplíen el rango de edad estudiado e incluyan muestras más amplias y heterogéneas con el fin de explorar el comportamiento de rechazo a la escuela en diferentes países. En este sentido, un estudio comparativo con grupos que procedan de culturas que tiendan hacia el individualismo permitiría contrastar las prioridades de los valores individuales y grupales en estudiantes que rechazan la escuela. Además, a pesar de que este estudio se ha llevado a cabo con muestra adolescente, al considerar este momento del desarrollo una fase en la que se producen tanto en cambios físicos como psicológicos con el fin de formar la identidad (Guadarrama, Mendoza, Márquez, Veytia y Padilla, 2018), se pretende que el análisis de esta conducta problemática se lleve a cabo también en edades más tempranas como la etapa de la escolaridad básica.

En segundo lugar, sería conveniente la realización de estudios longitudinales con el fin de analizar la evolución del comportamiento de rechazo a lo largo del tiempo. Por otro lado, a pesar de que en este estudio ha participado una muestra representativa de Ecuador y Chile con un total de 4266 participantes e indicando las poblaciones en las que se llevó a cabo la investigación, se espera que futuros trabajos incluyan, como información adicional a las características de la muestra, el contexto socioeconómico y cultural de los participantes con el fin de valorar, como variable de interés en futuros trabajos, si este factor puede condicionar las manifestaciones de rechazo escolar (Balfanz y Byrnes, 2012; Inglés et al., 2015; Kearney y Bates, 2005; Lyon, 2010).

En el análisis de los hallazgos encontrados cabe mencionar que estos datos deben ser interpretados con cautela ya que, tal y como señalan Estrada y Terpstra (2014), existen limitaciones que acompañan a los estudios transculturales en los que se comparan resultados en dos países. En este caso, se ha de 
valorar que este estudio tan solo incluye dos países de Latinoamérica, Ecuador y Chile, por lo que su generalización se limita a las poblaciones estudiadas, a pesar de que servirá de referencia para establecer futuras diferencias y similitudes respecto al rechazo escolar entre otros países latinoamericanos o estableciendo comparaciones respecto a países norteamericanos, europeos, asiáticos, etcétera.

Por último, debido a la ausencia de investigaciones previas que hayan puesto a prueba la formación de perfiles de rechazo mediante el método de conglomerados y utilizando la SRAS-R, no ha sido posible contrastar las evidencias encontradas con estudios previos. No obstante, la carencia de investigaciones en esta línea convierte los hallazgos del presente estudio en resultados pioneros en materia de perfiles de rechazo escolar ampliando, a su vez, el alcance de la investigación a un nuevo contexto sociocultural.

\section{Referencias bibliográficas}

Balfanz, R.y Byrnes, V. (2012). Chronic Absenteeism: Summarizing What We Know From Nationally Available Data. Baltimore: Johns Hopkins University Center for Social Organization of Schools.

Barry, A.E., Chaney, B.y Chaney, D. (2010). The impact of truant and alcohol-related behaviour on educational aspirations: a study of US high school Senior. Journal of School Health, 81(8), 485-492. http://dx.doi.org/10.1111/j.17461561.2011.00618.x

Blagg, N. (1987). School phobia and its treatment. Londres: Croom Helm.

Cohen, J. (1988). Statistical power analysis for the behavioral sciences (2nd ed.). Hillsdale, NJ: Erlbaum.

Dube, S.R. y Orpinas, P. (2009). Understanding excessive school absenteeism as school refusal behavior. Children \& Schools, 31(2), 87-95. http://dx.doi.org/10.1093/cs/31.2.87

Espinoza, O., González, L.E., Cruz, E.S. Castillo, D. y Loyola, J. (2014). Deserción escolar en Chile: un estudio de caso en relación con factores intraescolares. Educación y Educadores, 17(1), 32-50.

Estrada, S. y Terpstra, N. (2014). La complejidad de la variación transcultural: valores en Guatemala y Estados Unidos. Interamerican Journal of Psychology, 48(2), 152-165.

Fernández-Sogorb, A., Inglés, C.J., Sanmartín, R., Gonzálvez, C., Vicent, M. y García-Fernández, J.M. (2018). Validation of the Visual Analogue Scale for Anxiety-Revised and school refusal across anxiety profiles. International Journal of Clinical and Health Psychology, 18, 264-272. http://dx.doi.org/10.1016/j.ijchp.2018.07.002

García-Fernández, J.M., Inglés, C.J., Gonzálvez, C., Vicent, M., Delgado, B. y Gómez-Núñez, M.I. (2016). Revisión bibliométrica del rechazo escolar: perspectivas de investigación y su análisis. Educatio Siglo XXI, 34(1), 71-92. http://dx.doi.org/10.6018/j/252541

García-Fernández, J.M., Inglés, C.J., Lagos-San Martín, N., Gonzálvez, C., Vicent, M. y GómezNúñez, M.I. (2016). Diferencias de género y edad en rechazo escolar en una muestra de adolescentes chilenos. Estudios pedagógicos, $\operatorname{XLII}(1), 127-137$.

Gonzálvez, C., Inglés, C.J., Kearney, C.A., Vicent, M., Sanmartín, R. yGarcía-Fernández, J.M. (2016). School Refusal Assessment Scale-Revised: factorial invariance and latent means differences across gender and age in Spanish children. Frontiers in Psychology, 7, 1-10. http://dx.doi.org/10.3389/fpsyg.2016.02011

Gonzálvez, C., Inglés, C.J. yGarcía-Fernández, J.M. (2018).No quiero ir al colegio. El niño que rechaza la escuela. Madrid: Pirámide.

Gonzálvez, C., Sanmartín, R., Vicent, M., Inglés, C.J., Aparicio, M.P. y García-Fernández, J.M. (2018). Academic self-attributions for success and failure in mathematics and school refusal. Psychology in the Schools, 366-376. http:// dx.doi.org/10.1002/pits.22117

Guadarrama, R., Mendoza, V., Márquez, O., Veytia, M. y Padilla, I.M. (2018).Diferencias en la aplicación de estrategias de afrontamiento entre alumnos hombres y mujeres del nivel medio superior. Interdisciplinaria, 35, 205-2016.http://dx.doi.org/10.16888/ 
interd.2018.35.1.11

Haight, C., Kearney, C.A., Hendron, M. y Schafer, R. (2011).Confirmatory Analyses of the School Refusal Assessment Scale- Revised: Replication and Extension to a Truancy Sample. Journal Psychopathology Behavioral Assessment, 33(2), 196-204. http://dx.doi. org/10.1007/s10862-011-9218-9

Heyne, D., Vreeke, L.J., Maric, M., Boelens, H. y Van Widenfelt, B.M. (2016). Functional Assessment of School Attendance Problems: An Adapted Version of the School Refusal Assessment Scale-Revised. Journal of Emotional and Behavioral Disorders, 1-15. http://dx.doi.org/10.1177/1063426616661701

Inglés, C.J., Gonzálvez, C., García-Fernández, J.M., Vicent, M.y Martínez-Monteagudo, M.C. (2015). Current status of research on school refusal. European Journal of Education and Psychology, 8(1), 39-54.

Inglés, C.J., García-Fernández, J.M., Vicent, M., Gonzálvez, C. y Sanmartín, R. (2016). Profiles of Perfectionism and School Anxiety: A Review of the $2 \times 2$ Model of Dispositional Perfectionism in Child Population. Frontiers in Psychology, 7, 1-11. http://dx.doi.org/10.3389/ fpsyg.2016.01403.

Kashima, Y. (2016). Culture and psychology in the $21^{\text {st }}$ century: conceptions of culture and person for psychology revisited. Journal of Cross-Cultural Psychology, 47(1), 4-20. http:// dx.doi.org/10.1177/0022022115599445

Kearney, C.A. (2002). Identifying the function of school refusal behavior: A revision of the School Refusal Assessment Scale. Journal of Psychopathology and Behavioral Assessment, 24(4), 235-245. http://dx.doi. org/10.1023/A:1020774932043

Kearney, C.A. y Albano, A. (2004). The functional profiles of school refusal behavior: Diagnostic aspects. Behavior Modification, 28(1), 147-161. http://dx.doi. org/10.1177/01454455033259263.

Kearney, C.A.y Bates, M. (2005). Addressing school refusal behavior: Suggestions for frontline professionals. Children \& Schools, 27(4), 207-216. http://dx.doi.org/10.1093/cs/27.4.207
Kearney, C.A. (2006). Confirmatory factor analysis of the School Refusal Assessment Scale-Revised: Child and parent versions. Journal of Psychopathology and Behavioral Assessment, 28(3), 139-144. http://dx.doi. org/10.1007/s10862-005-9005-6

Kearney, C.A., Lemos, A.y Silverman, J. (2006). School refusal behavior. En R.B. Mennuti, A. Freeman y R.W. Christner (Eds.), Cognitive-behavioral interventions in educational settings: A handbook for practice (pp. 89-105). New York: Brunner-Routledge.

Kearney, C.A. y Albano, A. (2007). When children refuse school. A cognitive- behavioral therapy approach. Therapist Guide. Nueva York: Oxford University Press.

Kearney, C.A. (2008). School absenteeism and school refusal behavior in youth: A contemporary review. Clinical Psychology Review, 28(3), 451-471. http://dx.doi.org/10.1016/j. cpr.2007.07.012

Kearney, C.A. (2016). Managing school absenteeism at multiple tiers: an evidence-based and practical guide for professionals. New York: Oxford University Press.

King, N.J., Tonge, B.J., Heyne, D., Young, D., Myerson, N., Rollings, S., Pritchard, M.y Ollendick, T.H. (1998). Cognitive-behavioral treatment of school-refusing children: A controlled evaluation. Journal of the American Academy of Child and Adolescent Psychiatry, 37, 375-403.

Lyon, A.R. (2010). Confirmatory Factor Analysis of the School Refusal Assessment Scale-Revised in an African American Community Sample. Journal of Psychoeducational Assessment, 28(6), 511-523. http://dx.doi. org/10.1177/0734282909353438

Maynard, B.R., Salas-Wright, C.P., Vaughn, M.G. y Peters, K.E. (2012). Who are truant youth? Examining distinctive profiles of truant youth using latent profile analysis. Journal of Youth and Adolescence, 41(12), 1671-1684. http:// dx.doi.org/10.1007/s10964-012-9788-1

Moreno, D. (2013). La deserción escolar: un problema de carácter social. Revista In Vestigium Ire, 6, 115-124. 
Murrieta, P. (2016). Child Labor and Household Composition: Determinants of Child Labor in Mexico. Asian Journal of Latin American Studies, 29(3), 29-54.

Oyserman, D., Coon, H.M. y Kemmelmeier, M. (2002). Rethinking individualism and collectivism: evaluation of theoretical assumptions and meta-analyses. Psychological Bulletin, 128(1), 3-72. http://dx.doi.org/10.1037//00332909.128.1.3

Palomar, J. y Victorio, A. (2016). Expectativas laborales en la adolescencia: correlatos psicosociales. Interdisciplinaria, 33(1), 95-110. doi:10.16888/interd.2016.33.1.6

Pedraza, Z. (2007). El trabajo infantil en clave colonial: consideraciones histórico-antropológicas. Nómadas, 26, 80-90.

Post, D. (2011).Primary school student employment and academic achievement in Chile, Colombia, Ecuador and Peru. International Labour Review, 150(3-4), 255-278. http:// dx.doi.org/10.1111/j.1564-913X.2011.00116.x

Richards, H.J. y Hadwin, J.A. (2011).An exploration of the relationship between trait anxiety and school attendance in young people.School Mental Health, 3(4), 236-244. http://dx.doi. org/10.1007/s12310-011-9054-9

Seçer, I. (2014). The adaptation of School Refusal Assessment Scale into Turkish: Reliability and
Validity studies. Pakistan Journal of Statistics, 30(6), 1197-1202.

SITEAL (2010).Informe SITEAL 2010.Metaseducativas 2021: desafios y oportunidades. Buenos Aires: IIPE-UNESC.

Thornton, M., Darmody, M. y McCoy, S. (2013). Persistent absenteeism among Irish primary school pupils. Educational Review, 65(4), 488-501. http://dx.doi.org/10.1080/00131911. 2013.768599

Vicent, M., Inglés, C.J., Sanmartín, R., Gonzálvez, C. y García-Fernández, J.M. (2018). Aggression profiles in the Spanish child population: differences in perfectionism, school refusal and affect. Frontiers in Behavioral Neuroscience, 12, 1-12. http://dx.doi.org/10.3389/ fnbeh.2018.00012

Walter, D., Bialy, J.V., Wirth, E.V. y Doepfner, M. (2017).Psychometric Properties of the German School Refusal Assessment Scale-Revised. Journal of Psychoeducational Assessment, 1-5. http://dx.doi.org/10.1177/0734282916689641

Yahaya, A., Ramli, J., Hashim, S., Ibrahim, M.A., Kadir, H.B.H., Boon, Y. y Rahman, R.R.R. (2010). The effects of various modes of absenteeism problem in school on the academic performance of students in secondary schools. European Journal of Social Sciences, 12(4), 624-639. 
